\title{
Surfaces and Fronts with Harmonic-mean Curvature One in Hyperbolic Three-space
}

\author{
Masatoshi KOKUBU \\ Tokyo Denki University \\ (Communicated by M. Guest) \\ Dedicated to the memory of Professor Takao Sasai
}

\begin{abstract}
We show a global representation formula for a certain kind of Weingarten surface in hyperbolic three-space, which is based on the formula due to Gálvez, Martínez and Milán.

As an application of the representation formula, we also investigate surfaces with harmonic-mean curvature one (HMC-1 surfaces). We allow them to have certain kinds of singularities, and discuss some global properties.
\end{abstract}

\section{Introduction}

In the differential geometry of surfaces in hyperbolic three-space $\mathbf{H}^{3}$, surfaces with constant mean curvature one (CMC-1 surfaces, for short) are one of the central subjects [1], [10], [2], etc. The theory of flat surfaces in $\mathbf{H}^{3}$ is also developing, thanks to the appearance of a representation formula due to Gálvez, Martínez and Milán [4]. From the viewpoint of global theory for flat surfaces, one should generalize the category of surfaces to that of fronts. (Roughly speaking, a front is a surface with certain kinds of singularities.) Any complete flat surface in $\mathbf{H}^{3}$ must be a horosphere or a hyperbolic cylinder, however, many complete flat fronts exist in $\mathbf{H}^{3}$. (See [8].)

On the other hand, Gálvez, Martínez and Milán [5] also studied a wider class of surfaces in $\mathbf{H}^{3}$, including both CMC-1 surfaces and flat surfaces. It is the class of Weingarten surfaces satisfying $\alpha(H-1)=\beta K$ for some constants $\alpha$ and $\beta$. Here, $H$ denotes the mean curvature, and $K$ is the Gaussian curvature. A representation formula for such surfaces is shown in [5]. As a refinement of their formula, we shall prove the following theorem (Theorem 3.8):

THEOREM. Let $G$ be a meromorphic function on a Riemann surface M. Let $|\eta|^{2}$ be a Hermitian pseudometric on $M$ of constant curvature $\varepsilon$, and $h$ the developing map. Suppose that the quadratic differential form

$$
\frac{\left(1+\varepsilon|h|^{2}\right)^{2}}{4}|\{G ; h\} d h|^{2}-\frac{(1-\varepsilon)^{2}|d h|^{2}}{\left(1+\varepsilon|h|^{2}\right)^{2}}
$$

Received March 5, 2008

2000 Mathematics Subject Classification: 53C45 (Primary), 53A35 (Secondary) 
is definite on $M$. Then $f:=\mathcal{G H}^{*}: M \rightarrow \mathbf{H}^{3}=\mathrm{SL}(2, \mathbf{C}) / \mathrm{SU}(2)$, determined by

$$
\mathcal{G}:=\left(-G_{h}\right)^{-3 / 2}\left[\begin{array}{cc}
-G G_{h} & G G_{h h} / 2-G_{h}^{2} \\
-G_{h} & G_{h h} / 2
\end{array}\right] \text { and } \mathcal{H}:=\left[\begin{array}{cc}
\frac{1+\varepsilon^{2}|h|^{2}}{1+\varepsilon|h|^{2}} & \bar{h} \\
h & 1+\varepsilon|h|^{2}
\end{array}\right] \text {, }
$$

is a Weingarten immersion from $M$ to $\mathbf{H}^{3}$ with $2 \varepsilon(H-1)=(\varepsilon-1) K$.

Conversely, any Weingarten surface of $\alpha(H-1)=\beta K(\alpha \neq 2 \beta)$, except a horosphere, has this representation in terms of $(G, h)$.

Here $\{G ; h\}$ denotes the Schwarzian derivative of $G$ with respect to $h$, and $G_{h}=d G / d h$, $G_{h h}=d^{2} G / d h^{2}$.

The theorem above is a global formula in contrast to the formula due to Gálvez, Martínez and Milán.

For the ratio $[\alpha: \beta]$ in $\mathbf{R} P^{1}$, let $\mathcal{W}_{[\alpha: \beta]}$ be the set of Weingarten surfaces satisfying $\alpha(H-1)=\beta K$, and set

$$
\mathcal{W}:=\bigcup_{[\alpha: \beta] \in \mathbf{R} P^{1}} \mathcal{W}_{[\alpha: \beta]}
$$

It is remarkable that $\mathcal{W}$ is closed under parallel transforms, that is, any parallel surface of any surface in $\mathcal{W}$ is always in $\mathcal{W}$. More precisely, dividing $\mathcal{W}$ into four subclasses

$$
\mathcal{W}^{0}:=\mathcal{W}_{[0: 1]}, \quad \mathcal{W}^{1}:=\bigcup_{\lambda<1 / 2} \mathcal{W}_{[1: \lambda]}, \quad \mathcal{W}^{2}:=\mathcal{W}_{[1: 1 / 2]}, \quad \mathcal{W}^{3}:=\bigcup_{\lambda>1 / 2} \mathcal{W}_{[1: \lambda]},
$$

we can prove that each $\mathcal{W}^{j}$ is closed under parallel transforms. (See Theorem 2.3 and Theorem 2.5.) Hence, roughly speaking, we can say that CMC-1 surface theory represents the theory of surfaces in $\mathcal{W}^{1}$. For instance, one can construct a Weingarten surface satisfying $H-1=\lambda K(\lambda<1 / 2)$, though it may have singularities, by constructing any CMC-1 surface first and by parallel transforming it appropriately. By the same reasoning, the theory of surfaces in $\mathcal{W}^{3}$ can be represented by one special type of surface. We will take $\mathcal{W}_{[1: 1]}$ as such a representative for $\mathcal{W}^{3}$, because surfaces in $\mathcal{W}_{[1: 1]}$, i.e., Weingarten surfaces satisfying $H-1=K$, have another special geometric meaning: the sum of the reciprocals of the principal curvatures is 2 . In other words, the harmonic mean of the principal curvature functions is 1. We also call them surfaces with constant harmonic-mean curvature one (HMC-1 surfaces, for short).

For the reason mentioned above, we will study HMC-1 surfaces in this paper. Although much work has been done on CMC-1 surfaces, HMC-1 surfaces have received less attention. For example, there is Epstein's work [3], however, it seems lesser-known. (In classical Euclidean surface theory, the radii of principal curvature were considered as the fundamental entities. There is some work on the mean radius of principal curvatures, or equivalently, about the harmonic mean of principal curvatures; e.g., Christoffel's theorem about rigidity of surfaces (cf. [9, pp. 299-302]).) 
In Section 2, we discuss background material for the sake of precisely understanding the topics mentioned in this introduction. Section 3 is devoted to deriving the formula due to Gálvez, Martínez and Milán, and its global version. HMC-1 surfaces are treated in Section 4. As for the case of flat surfaces, it is more natural to consider HMC-1 fronts rather than HMC-1 surfaces. HMC-1 fronts are defined in Section 5.

The author would like to thank Professors Wayne Rossman, Masaaki Umehara and Kotaro Yamada for their valuable comments.

\section{Background}

2.1. Basics. Let $\mathbf{L}^{4}$ denote the Minkowski 4 -space with the Lorentzian inner product $\langle,\rangle_{L}$ of signature $(-,+,+,+)$. Let $\mathcal{F}$ be the set of positively oriented and positively timeoriented frames $\left(e_{0}, e_{1}, e_{2}, e_{3}\right)$ in $\mathbf{L}^{4}$ satisfying

$$
\left\langle e_{\alpha}, e_{\beta}\right\rangle_{L}= \begin{cases}-1 & \text { if } \alpha=\beta=0, \\ 0 & \text { if } \alpha \neq \beta, \\ 1 & \text { if } \alpha=\beta>0 .\end{cases}
$$

The indices $\alpha$ and $\beta$ run over $0,1,2,3$, while the indices $i, j$ and $k$ run over $1,2,3$. We shall use Einstein's convention, that is, the symbol $\sum$ may be omitted for sums over repeated indices.

Regarding $e_{\alpha}:\left(e_{0}, e_{1}, e_{2}, e_{3}\right) \ni \mathcal{F} \mapsto e_{\alpha} \in \mathbf{L}^{4}(\alpha=0,1,2,3)$ as $\mathbf{L}^{4}$-valued functions, $d e_{\alpha}(\alpha=0,1,2,3)$ are $\mathbf{L}^{4}$-valued one-forms on $\mathcal{F}$. The connection forms $\omega_{\alpha}^{\beta}$ are defined by $d e_{\alpha}=e_{\beta} \otimes \omega_{\alpha}^{\beta}$. We write $\omega^{i}$ for $\omega_{0}^{i}$. Differentiating (2.1), we have

$$
\begin{aligned}
& \omega_{\alpha}^{\alpha}=0, \quad-\omega_{i}^{0}+\omega_{0}^{i}=0, \quad \omega_{i}^{j}+\omega_{j}^{i}=0, \\
& d e_{0}=e_{i} \otimes \omega^{i}, \quad d e_{i}=e_{0} \otimes \omega^{i}+e_{j} \otimes \omega_{i}^{j} .
\end{aligned}
$$

Again, differentiating (2.3), we have the structure equations:

$$
d \omega^{i}=-\omega_{j}^{i} \wedge \omega^{j}, \quad d \omega_{j}^{i}=-\omega_{k}^{i} \wedge \omega_{j}^{k}-\omega^{i} \wedge \omega^{j} .
$$

The hyperbolic 3-space $\mathbf{H}^{3}$ is the upper half component of the two-sheeted hyperboloid in $\mathbf{L}^{4}$, i.e.,

$$
\mathbf{H}^{3}=\left\{x=\left(x_{0}, x_{1}, x_{2}, x_{3}\right) \in \mathbf{L}^{4} ;\langle x, x\rangle_{L}=-1, x_{0}>0\right\}
$$

with the metric induced by $\langle,\rangle_{L}$. $\mathbf{H}^{3}$ is a space form of constant negative curvature -1 . As usual, we regard $e_{0}: \mathcal{F} \rightarrow \mathbf{H}^{3} \subset \mathbf{L}^{4}$ as the oriented orthonormal frame bundle of $\mathbf{H}^{3}$.

Let $M$ be a connected, oriented surface, and $f: M \rightarrow \mathbf{H}^{3}$ an immersion. Let $\left\{\varepsilon_{1}, \varepsilon_{2}\right\}$ be a local orthonormal frame on $U \subset M$, and let $v$ denote a unit normal field. Regarding them 
as $\mathbf{L}^{4}$-valued functions, we consider a map

$$
\left(e_{0}, e_{1}, e_{2}, e_{3}\right):=\left(f, \varepsilon_{1}, \varepsilon_{2}, v\right): U \rightarrow \mathcal{F} .
$$

We shall use the same notation for differential forms on $\mathcal{F}$ and forms on $U$ pulled back by this map. Since $\langle v, d f\rangle_{L}=0$,

$$
0=\langle\nu, d f\rangle_{L}=\left\langle e_{3}, d e_{0}\right\rangle_{L}=\omega^{3} .
$$

From now on, we shall use the following convention on the ranges of indices: $1 \leq i, j, k \leq 2$. It follows from (2.2), (2.3) and (2.5) that

$$
\begin{aligned}
& \omega_{\alpha}^{\alpha}=0, \quad-\omega_{i}^{0}+\omega_{0}^{i}=0, \quad-\omega_{3}^{0}+\omega_{0}^{3}=0, \quad \omega_{i}^{j}+\omega_{j}^{i}=0, \quad \omega_{3}^{j}+\omega_{j}^{3}=0, \\
& d e_{0}=e_{i} \otimes \omega^{i}, \quad d e_{i}=e_{0} \otimes \omega^{i}+e_{j} \otimes \omega_{i}^{j}+e_{3} \otimes \omega_{i}^{3}, \quad d e_{3}=e_{j} \otimes \omega_{3}^{j} .
\end{aligned}
$$

The structure equations (2.4) become

$$
\begin{aligned}
& d \omega^{i}=-\omega_{j}^{i} \wedge \omega^{j}, \quad 0=d \omega^{3}=-\omega_{j}^{3} \wedge \omega^{j}, \\
& d \omega_{2}^{1}=-\omega_{3}^{1} \wedge \omega_{2}^{3}-\omega^{1} \wedge \omega^{2}, \quad d \omega_{j}^{3}=-\omega_{k}^{3} \wedge \omega_{j}^{k} .
\end{aligned}
$$

Following Bryant's notation ([1]), we introduce two complex-valued one-forms $\omega:=\omega^{1}+$ $\sqrt{-1} \omega^{2}, \pi:=\omega_{1}^{3}-\sqrt{-1} \omega_{2}^{3}$, and a complex vector $e:=\left(e_{1}-\sqrt{-1} e_{2}\right) / 2$. Then (2.7) and (2.8) are rewritten as

$$
\begin{aligned}
d \omega & =\sqrt{-1} \omega_{2}^{1} \wedge \omega, \quad \omega \wedge \pi+\bar{\omega} \wedge \bar{\pi}=0, \\
d \omega_{2}^{1} & =-\frac{\sqrt{-1}}{2}(\pi \wedge \bar{\pi}+\omega \wedge \bar{\omega}), \quad d \pi=-\sqrt{-1} \omega_{2}^{1} \wedge \pi .
\end{aligned}
$$

The first fundamental form $\mathrm{I}=\left\langle d e_{0}, d e_{0}\right\rangle_{L}$ is given by

$$
\mathrm{I}=\left\langle e_{i} \otimes \omega^{i}, e_{i} \otimes \omega^{i}\right\rangle_{L}=\left(\omega^{1}\right)^{2}+\left(\omega^{2}\right)^{2}=\omega \bar{\omega}=|\omega|^{2} .
$$

The Gaussian curvature $K$ is determined by $d \omega_{2}^{1}=K(\sqrt{-1} / 2) \omega \wedge \bar{\omega}$. Hence, it follows from (2.10) that

$$
(K+1) \omega \wedge \bar{\omega}+\pi \wedge \bar{\pi}=0 .
$$

The second fundamental form $\mathbb{I}=-\left\langle d e_{0}, d e_{3}\right\rangle_{L}$ is given by

$$
\mathbf{I}=-\left\langle e_{i} \otimes \omega^{i}, e_{i} \otimes \omega_{3}^{i}\right\rangle_{L}=\omega^{1} \omega_{1}^{3}+\omega^{2} \omega_{2}^{3}=\frac{1}{2}(\omega \pi+\bar{\omega} \bar{\pi})=\operatorname{Re}(\omega \pi) .
$$

If we set $\omega_{i}^{3}=h_{i j} \omega^{j}$, then $h_{12}=h_{21}$ and $\mathbb{I}=h_{11}\left(\omega^{1}\right)^{2}+2 h_{12} \omega^{1} \omega^{2}+h_{22}\left(\omega^{2}\right)^{2}$. Moreover,

$$
\pi=\frac{1}{2}\left\{\left(h_{11}-h_{22}\right)-2 \sqrt{-1} h_{12}\right\} \omega+\frac{1}{2}\left(h_{11}+h_{22}\right) \bar{\omega} .
$$

Setting $q=\left\{\left(h_{11}-h_{22}\right)-2 i h_{12}\right\} / 2$ and $H=\left(h_{11}+h_{22}\right) / 2$, we can write

$$
\pi=q \omega+H \bar{\omega} .
$$


Here, $H$ is the mean curvature. The second fundamental form II is written as

$$
\mathrm{II}=\frac{q}{2} \omega \omega+H \omega \bar{\omega}+\frac{\bar{q}}{2} \bar{\omega} \bar{\omega} .
$$

It follows from (2.11) and (2.12) that

$$
K=-1+H^{2}-|q|^{2}\left(=-1+\operatorname{det}\left(h_{i j}\right)\right) .
$$

As a corollary,

$$
H^{2}-K-1 \geq 0
$$

holds at every point $p \in M$, with equality if and only if $p$ is an umbilic point.

The third fundamental form III $=\left\langle d e_{3}, d e_{3}\right\rangle_{L}$ is given by

$$
\text { III }=\left\langle e_{i} \otimes \omega_{3}^{i}, e_{i} \otimes \omega_{3}^{i}\right\rangle_{L}=\left(\omega_{3}^{1}\right)^{2}+\left(\omega_{3}^{2}\right)^{2}=\pi \bar{\pi}=|\pi|^{2} .
$$

The ideal boundary $\partial \mathbf{H}^{3}$ is considered as the quotient space $N^{3} / \sim$, where

$$
N^{3}=\left\{x=\left(x_{0}, x_{1}, x_{2}, x_{3}\right) \in \mathbf{L}^{4} ;\langle x, x\rangle_{L}=0, x_{0}>0\right\}
$$

and $x \sim y$ if $x=\lambda y$ for some positive constant $\lambda$. In other words, $\partial \mathbf{H}^{3}$ consists of positive null half-lines in $\mathbf{L}^{4}$. $N^{3} / \sim$ is diffeomorphic to the 2-sphere, and a natural conformal structure on $N^{3} / \sim$ is given by the induced metric on $N^{3}$. Hence, $\partial \mathbf{H}^{3}\left(=N^{3} / \sim\right)$ is identified with the conformal 2-sphere. By definition, the hyperbolic Gauss maps are $G^{ \pm}=\left[e_{0} \pm e_{3}\right]: M \rightarrow$ $\partial \mathbf{H}^{3}$, where $[v]$ denotes the line spanned by $v \in \mathbf{L}^{4}$. Because we mainly treat $G^{+}=\left[e_{0}+e_{3}\right]$, we simply write $G$ for $G^{+}$.

The conformal structure on $M$ induced by $G$ is the conformal class determined by $\left\langle d\left(e_{0}+\right.\right.$ $\left.\left.e_{3}\right), d\left(e_{0}+e_{3}\right)\right\rangle_{L}$. Indeed, it is computed as follows:

LEMMA 2.1.

$$
\left\langle d\left(e_{0}+e_{3}\right), d\left(e_{0}+e_{3}\right)\right\rangle_{L}=|\omega-\bar{\pi}|^{2}=2(H-1) \mathbb{I}-K \mathrm{I} .
$$

PROOF. Since $d e_{0}+d e_{3}=e \otimes(\omega-\bar{\pi})+\bar{e} \otimes(\bar{\omega}-\pi)$ holds by (2.6), the first equality is obvious. The second equality follows from a straightforward computation using (2.12), (2.13) and (2.14).

Similarly, the third fundamental form $\mathrm{III}=\left\langle d e_{3}, d e_{3}\right\rangle_{L}$ is computed as

$$
\mathrm{III}=\left\langle d e_{3}, d e_{3}\right\rangle_{L}=2 H \mathrm{II}-(K+1) \mathrm{I} .
$$

Proposition 2.2. The Gaussian curvature $\tilde{K}$ of the pseudometric $\left\langle d\left(e_{0}+e_{3}\right), d\left(e_{0}+\right.\right.$ $\left.\left.e_{3}\right)\right\rangle_{L}$ is

$$
\tilde{K}=\frac{K}{K-2(H-1)}
$$


Proof. Setting $\alpha=\omega-\bar{\pi}$, we have

$$
d \alpha=d \omega-d \bar{\pi}=\sqrt{-1} \omega_{2}^{1} \wedge \omega-\left(\sqrt{-1} \omega_{2}^{1} \wedge \bar{\pi}\right)=\sqrt{-1} \omega_{2}^{1} \wedge \alpha .
$$

Hence, we can consider $\omega_{2}^{1}$ as the connection form of the metric $|\alpha|^{2}$. On the other hand, by (2.12) and (2.14),

$$
\begin{aligned}
\alpha \wedge \bar{\alpha} & =(\omega-\bar{\pi}) \wedge(\bar{\omega}-\pi)=\{(1-H) \omega-\bar{q} \bar{\omega})\} \wedge\{(1-H) \bar{\omega}-q \omega)\} \\
& =\left\{(1-H)^{2}-|q|^{2}\right\} \omega \wedge \bar{\omega}=(K-2 H+2) \omega \wedge \bar{\omega} .
\end{aligned}
$$

Therefore

$$
d \omega_{2}^{1}=K \cdot \frac{\sqrt{-1}}{2} \omega \wedge \bar{\omega}=\frac{K}{K-2 H+2} \cdot \frac{\sqrt{-1}}{2} \alpha \wedge \bar{\alpha},
$$

which proves the assertion.

2.2. Parallel surfaces. The map $f_{t}:=\cosh t f+\sinh t v$ is called the parallel surface of $f$ at distance $t$. It is easily verified that $f_{t}: M \rightarrow \mathbf{H}^{3}$ and that $f_{t}(p)$ is joined to $f(p)$ by a hyperbolic line segment of length $t$. In general, $f_{t}$ may fail to be an immersion. In fact, $f_{t}$ is an immersion if and only if $\cosh t \omega^{i}+\sinh t \omega_{3}^{i} \neq 0$ for every $p \in M$, because

$$
\begin{aligned}
d f_{t} & =\cosh t d f+\sinh t d \nu=\cosh t d e_{0}+\sinh t d e_{3} \\
& =\cosh t e_{i} \otimes \omega^{i}+\sinh t e_{i} \otimes \omega_{3}^{i}=e_{i} \otimes\left(\cosh t \omega^{i}+\sinh t \omega_{3}^{i}\right) .
\end{aligned}
$$

In this section, we assume that $f_{t}$ is an immersion, unless otherwise stated.

The first fundamental form $\mathrm{I}_{t}=\left\langle d f_{t}, d f_{t}\right\rangle_{L}$ is

$$
\mathrm{I}_{t}=\left(\cosh t \omega^{1}+\sinh t \omega_{3}^{1}\right)^{2}+\left(\cosh t \omega^{2}+\sinh t \omega_{3}^{2}\right)^{2},
$$

hence, the $\theta^{i}:=\cosh t \omega^{i}+\sinh t \omega_{3}^{i}(i=1,2)$ form an orthonormal frame of $f_{t}$. It follows from the structure equations (2.7) and (2.8) that

$$
d \theta^{i}=-\omega_{j}^{i} \wedge \theta^{j}
$$

Thus $\omega_{2}^{1}$ is also a connection form of $\mathrm{I}_{t}=\left(\theta^{1}\right)^{2}+\left(\theta^{2}\right)^{2}$. Denoting the Gaussian curvature of I $t$ by $K_{t}$, we have

$$
d \omega_{2}^{1}=K_{t} \theta^{1} \wedge \theta^{2}\left(=K \omega^{1} \wedge \omega^{2}\right) .
$$

Using $K=-1+\operatorname{det}\left(h_{i j}\right)$ and $2 H=h_{11}+h_{22}$, we have

$$
\theta^{1} \wedge \theta^{2}=\left\{\cosh ^{2} t-2 H \cosh t \sinh t+(K+1) \sinh ^{2} t\right\} \omega^{1} \wedge \omega^{2} .
$$

Therefore

$$
d \omega_{2}^{1}=\frac{K}{K \sinh ^{2} t-2 H \cosh t \sinh t+\cosh ^{2} t+\sinh ^{2} t} \theta^{1} \wedge \theta^{2} .
$$


This implies that

$$
K_{t}=\frac{K}{K \sinh ^{2} t-2 H \cosh t \sinh t+\cosh ^{2} t+\sinh ^{2} t} .
$$

Thus, since $\left(K_{t}\right)_{-t}=K$, we have

$$
\frac{K_{t}}{K_{t} \sinh ^{2} t+2 H_{t} \cosh t \sinh t+\cosh ^{2} t+\sinh ^{2} t}=K .
$$

This formula together with (2.17) implies that

$$
H_{t}=\frac{H\left(\cosh ^{2} t+\sinh ^{2} t\right)-(2+K) \cosh t \sinh t}{K \sinh ^{2} t-2 H \cosh t \sinh t+\cosh ^{2} t+\sinh ^{2} t} .
$$

The formulas (2.17) and (2.18) yield the following well-known theorem:

THEOREM 2.3. (1) All parallel surfaces of a flat surface are also flat.

(2) A family of parallel surfaces of a surface with constant mean curvature $(|H|>1)$ contains a surface with constant Gaussian curvature $(K>0)$, and vice versa.

We can rewrite $(2.18)$ as

$$
H_{t}-1=\frac{(\cosh t+\sinh t)\{(\cosh t+\sinh t)(H-1)-K \sinh t\}}{K \sinh ^{2} t-2(H-1) \cosh t \sinh t+(\cosh t-\sinh t)^{2}} .
$$

Multiplying by $K$ on both sides, we have

$$
K\left(H_{t}-1\right)=K_{t} e^{t}\left\{e^{t}(H-1)-K \sinh t\right\} .
$$

For example, if we assume that the original surface $f$ has constant mean curvature one (CMC1), then

$$
H_{t}-1=\left(-e^{t} \sinh t\right) K_{t},
$$

thus, $f_{t}$ is a Weingarten surface. The family of Weingarten surfaces satisfying $H-1=\lambda K$ for some constant $\lambda$ includes the following interesting surfaces:

If $\lambda=0$, then $f$ is a CMC- 1 surface.

If $\lambda=1 / 2$, then at least one of the principal curvatures equals 1 .

If $\lambda=1$, then the sum of the reciprocals of the principal curvatures is 2 , that

is, $f$ is a surface with constant harmonic-mean curvature one (HMC-1).

These follow from $K=-1+\kappa_{1} \kappa_{2}, 2 H=\kappa_{1}+\kappa_{2}$ and the fact that the harmonic-mean curvature is $2 /\left(\kappa_{1}^{-1}+\kappa_{2}^{-1}\right)$, where $\kappa_{i}(i=1,2)$ denote the principal curvatures.

Conversely, let us assume that the original surface $f$ satisfies $H-1=\lambda K$ for some constant $\lambda$. Then the parallel surface $f_{t}$ satisfies

$$
H_{t}-1=\frac{(2 \lambda-1) e^{2 t}+1}{2} K_{t}
$$

and hence is the same kind of Weingarten surface. Since $\lambda_{t}:=\left\{(2 \lambda-1) e^{2 t}+1\right\} / 2$ satisfies $\left(2 \lambda_{t}-1\right)=(2 \lambda-1) e^{2 t}$, the following lemma is clear: 
LEMMA 2.4. (1) If $\lambda=1 / 2$, then $\lambda_{t}=1 / 2$ for all $t$.

(2) If $\lambda<1 / 2$, then $\lambda_{t}<1 / 2$ for all $t$, and $\lambda_{t}=0$ for some unique $t$.

(3) If $\lambda>1 / 2$, then $\lambda_{t}>1 / 2$ for all $t$, and $\lambda_{t}=1$ for some unique $t$.

Therefore, we have the following theorem:

THEOREM 2.5. (1) Let $f$ be a Weingarten surface such that at least one of the principal curvatures equals 1 , i.e., a Weingarten surface with $H-1=K / 2$. Then, for all parallel surfaces of $f$, at least one of the principal curvatures equals 1.

(2) Let $f$ be a Weingarten surface with $H-1=\lambda K$ for some constant $\lambda(>1 / 2)$. Then the family of parallel surfaces of $f$ consists of Weingarten surfaces with $H-1=\lambda K(\lambda>$ 1/2). This family includes a single HMC-1 surface.

(3) Let $f$ be a Weingarten surface with $H-1=\lambda K$ for some constant $\lambda(<1 / 2)$. Then the family of parallel surfaces of $f$ consists of Weingarten surfaces with $H-1=\lambda K(\lambda<$ 1/2). This family includes a single CMC-1 surface.

Theorem 2.3 is well-known, whereas Theorem 2.5 seems to be lesser-known.

2.3. Weingarten surfaces satisfying $\alpha(H-1)=\beta K$. Throughout this section, $f: M \rightarrow \mathbf{H}^{3}$ denotes a Weingarten surface satisfying $\alpha(H-1)=\beta K$ for some constants $\alpha$, $\beta$.

It follows from Lemma 2.1 that

$$
\begin{aligned}
\left\langle d e_{0}+d e_{3}, d e_{0}+d e_{3}\right\rangle_{L} & =2(H-1) \mathrm{II}-K \mathrm{I} \\
& = \begin{cases}-\frac{K}{\alpha}(\alpha \mathrm{I}-2 \beta \text { II }) & (\text { if } \alpha \neq 0) \\
\frac{1-H}{\beta}(\alpha \mathrm{I}-2 \beta \text { II }) & (\text { if } \beta \neq 0)\end{cases}
\end{aligned}
$$

Hence, if we endow $M$ with the "metric" $\alpha \mathrm{I}-2 \beta$ II, then the hyperbolic Gauss map $G$ is conformal. However, we need to check that $\alpha \mathrm{I}-2 \beta$ II is indeed a metric:

LEMMA 2.6. If $\alpha \neq 2 \beta$, then $\alpha \mathrm{I}-2 \beta$ II is either positive or negative definite.

ProOF. $\alpha \mathrm{I}-2 \beta \mathrm{II}$ is definite if and only if

$$
\operatorname{det}\left(\begin{array}{cc}
\alpha-2 \beta \lambda h_{11} & -2 \beta h_{12} \\
-2 \beta h_{12} & \alpha-2 \beta h_{22}
\end{array}\right)>0
$$

This condition (2.20) is equivalent to $\alpha^{2}-4 \alpha \beta H+4 \beta^{2}(K+1)>0$. Moreover, from the assumption $\alpha(H-1)=\beta K$, this is equivalent to $(\alpha-2 \beta)^{2}>0$.

Proposition 2.7. Let $f: M \rightarrow \mathbf{H}^{3}$ be a Weingarten surface satisfying $\alpha(H-1)=$ $\beta K$ for some constants $\alpha, \beta(\alpha \neq 2 \beta)$. Then $\alpha \mathrm{I}-2 \beta$ II determines a conformal structure on $M$, and the hyperbolic Gauss map $G:(M, \alpha \mathrm{I}-2 \beta \mathbf{I}) \rightarrow \partial \mathbf{H}^{3}$ is conformal.

Conversely, if an immersed surface $f: M \rightarrow \mathbf{H}^{3}$ satisfies

(i) $\quad \alpha_{0} \mathrm{I}-2 \beta_{0}$ II is definite, and 
(ii) $\quad G:\left(M, \alpha_{0} \mathrm{I}-2 \beta_{0} \mathbf{I}\right) \rightarrow \partial \mathbf{H}^{3}$ is conformal

for some constants $\alpha_{0}, \beta_{0}$, then $f$ is a totally umbilical surface or a Weingarten surface satisfying $\alpha_{0}(H-1)=\beta_{0} K$.

The preceding proposition is shown in [1] when $\beta=0$, and in [4] when $\alpha=0$. Moreover, the former part is proved in [5]. Hence we have only to prove the latter part. However, it can be proved by the same argument as in [1]. Thus, the proof is omitted here.

The following proposition follows immediately from Proposition 2.2.

PROPOSITION 2.8. For a Weingarten surface satisfying $\alpha(H-1)=\beta K(\alpha \neq 2 \beta)$, the pseudometric $\left\langle d\left(e_{0}+e_{3}\right), d\left(e_{0}+e_{3}\right)\right\rangle_{L}$ has constant curvature $\alpha /(\alpha-2 \beta)$.

From now on, we will assume $\alpha \neq 2 \beta$. We wish to consider $M$ a Riemann surface compatible with the conformal structure $[\alpha \mathrm{I}-2 \beta \mathrm{II}]$ providing an orientation. Following Bryant's notation ([1]), we set

$$
\eta:=\left(\omega^{1}-\omega_{1}^{3}\right)-\sqrt{-1}\left(\omega^{2}-\omega_{2}^{3}\right)(=\bar{\omega}-\pi) .
$$

Since $\left\langle d e_{0}+d e_{3}, d e_{0}+d e_{3}\right\rangle_{L}=|\eta|^{2}$, it follows from (2.19) that $|\eta|^{2}$ is (weakly) conformally equivalent to $\alpha \mathrm{I}-2 \beta$ II. Hence, we can give a complex structure on $M$ so that $\eta$ is a $(1,0)$ form. The following lemma is the key to the Gálvez-Martínez-Milán formula which will be discussed in the next section.

LEMMA 2.9.

$$
\bar{\partial} \partial\left(e_{0}+e_{3}\right)=\frac{1}{2} \frac{1}{\alpha-2 \beta}\left\{\beta e_{0}+(\alpha-\beta) e_{3}\right\} \otimes \eta \wedge \bar{\eta},
$$

that is,

$$
\left(e_{0}+e_{3}\right)_{z \bar{z}}=\frac{1}{2} \frac{|\hat{\eta}|^{2}}{2 \beta-\alpha}\left\{\beta e_{0}+(\alpha-\beta) e_{3}\right\}
$$

where $z$ is a local complex coordinate and $\eta=\hat{\eta} d z$.

PROOF. Because $d\left(e_{0}+e_{3}\right)=e \otimes \bar{\eta}+\bar{e} \otimes \eta$, we have $\partial\left(e_{0}+e_{3}\right)=\bar{e} \otimes \eta$. Furthermore, taking $\bar{\partial}$, we can calculate as follows:

$$
\begin{aligned}
\bar{\partial} \partial\left(e_{0}+e_{3}\right) & =\bar{\partial} \bar{e} \wedge \eta+\bar{e} \otimes \bar{\partial} \eta \\
& =d \bar{e} \wedge \eta+\bar{e} \otimes d \eta\left(\because \eta \in A^{1,0}\right) \\
& =\left\{e_{0} \otimes \frac{1}{2} \omega \wedge \eta+\sqrt{-1} \bar{e} \otimes \omega_{2}^{1} \wedge \eta+e_{3} \otimes \frac{1}{2} \bar{\pi} \wedge \eta\right\}+\bar{e} \otimes d \eta \\
& =e_{0} \otimes \frac{1}{2} \omega \wedge \eta+e_{3} \otimes \frac{1}{2} \bar{\pi} \wedge \eta .
\end{aligned}
$$

On the other hand, using (2.12), (2.14), we can compute $\omega \wedge \eta, \bar{\pi} \wedge \eta, \eta \wedge \bar{\eta}$ as follows:

$$
\omega \wedge \eta=\omega \wedge \bar{\omega}-\omega \wedge \pi=\omega \wedge \bar{\omega}-H \omega \wedge \bar{\omega}=(1-H) \omega \wedge \bar{\omega},
$$




$$
\begin{aligned}
& \bar{\pi} \wedge \eta=\bar{\pi} \wedge \bar{\omega}-\bar{\pi} \wedge \pi=-H \bar{\omega} \wedge \omega-(K+1) \omega \wedge \bar{\omega}=(H-K-1) \omega \wedge \bar{\omega} \\
& \eta \wedge \bar{\eta}=(-K-2+2 H) \omega \wedge \bar{\omega} .
\end{aligned}
$$

Moreover, from the condition $\alpha(H-1)=\beta K$, we have the following:

$$
\begin{aligned}
& \text { If } \alpha \neq 0, \quad \omega \wedge \eta=(1-H) \omega \wedge \bar{\omega}=-(\beta / \alpha) K \omega \wedge \bar{\omega}, \\
& \bar{\pi} \wedge \eta=\{(H-1)-K\} \omega \wedge \bar{\omega}=((\beta-\alpha) / \alpha) K \omega \wedge \bar{\omega}, \\
& \eta \wedge \bar{\eta}=\{-1+2(H-1)\} \omega \wedge \bar{\omega}=((2 \beta-\alpha) / \alpha) K \omega \wedge \bar{\omega} . \\
& \text { If } \alpha=0, \omega \wedge \eta=(1-H) \omega \wedge \bar{\omega}, \\
& \bar{\pi} \wedge \eta=(H-1) \omega \wedge \bar{\omega}, \\
& \eta \wedge \bar{\eta}=(-2+2 H) \omega \wedge \bar{\omega} .
\end{aligned}
$$

Substituting these into (2.21) completes the proof.

\section{The Gálvez-Martínez-Milán formula and its global version}

3.1. An overview on the work by Gálvez, Martínez and Milán. In this section we give an overview on deriving the Gálvez-Martínez-Milán formula.

First of all, we review the matrix model for $\mathbf{H}^{3}$. We identify $\mathbf{L}^{4}$ with $\operatorname{Herm}(2)$, the set of $2 \times 2$ Hermitian matrices, via

$$
\mathbf{L}^{4} \ni x=\left(x_{0}, x_{1}, x_{2}, x_{3}\right) \leftrightarrow X=\left[\begin{array}{cc}
x_{0}+x_{3} & x_{1}+\sqrt{-1} x_{2} \\
x_{1}-\sqrt{-1} x_{2} & x_{0}-x_{3}
\end{array}\right] \in \operatorname{Herm}(2) .
$$

Since $\langle x, x\rangle_{L}=-\operatorname{det} X$ and $2 x_{0}=\operatorname{tr} X$,

$$
\begin{aligned}
\mathbf{H}^{3} & =\{X \in \operatorname{Herm}(2) ; \operatorname{det} X=1, \operatorname{tr} X>0\} \\
& =\left\{a a^{*} ; a \in \operatorname{SL}(2, \mathbf{C})\right\}=\operatorname{SL}(2, \mathbf{C}) / \operatorname{SU}(2), \\
\partial \mathbf{H}^{3} & =\{X \in \operatorname{Herm}(2) ; \operatorname{det} X=0, \operatorname{tr} X>0\} / \sim \\
& =\left\{a a^{*} ; a=\left[\begin{array}{l}
a_{1} \\
a_{2}
\end{array}\right] \in \mathbf{C}^{2} \backslash\{0\}\right\} / \sim \\
& =\left(\mathbf{C}^{2} \backslash\{0\}\right) /(\mathbf{C} \backslash\{0\})=\mathbf{C} P^{1},
\end{aligned}
$$

where $a^{*}$ is the conjugate transpose of $a$, and $\mathbf{C} P^{1}$ is the complex projective line.

Hereafter, we will consider $\mathbf{H}^{3}$ to be $\operatorname{SL}(2, \mathbf{C}) / \mathrm{SU}(2)$, and $\partial \mathbf{H}^{3}$ to be $\mathbf{C} P^{1}$.

Recall that $G=\left[e_{0}+e_{3}\right]: M \rightarrow \mathbf{C} P^{1}\left(=\partial \mathbf{H}^{3}\right)$ is a conformal map. Thus

$$
e_{0}+e_{3}=\Lambda\left[\begin{array}{l}
A \\
B
\end{array}\right]\left[\begin{array}{ll}
\bar{A} & \bar{B}
\end{array}\right]
$$


for some holomorphic functions $A, B$ and a positive function $\Lambda$. Note that $A, B$ and $\Lambda$ have an ambiguity, but $\Lambda|A|^{2}, \Lambda|B|^{2}$ and $\Lambda A \bar{B}$ are well-defined on $M$.

Let $z$ be a local holomorphic coordinate on $M$, and let the lower suffix denote the partial derivative. Differentiating (3.1) twice, we have

$$
\left(e_{0}+e_{3}\right)_{z \bar{z}}=\left[\begin{array}{cc}
A & A_{z} \\
B & B_{z}
\end{array}\right]\left[\begin{array}{cc}
\Lambda_{z \bar{z}} & \Lambda_{z} \\
\Lambda_{\bar{z}} & \Lambda
\end{array}\right]\left[\begin{array}{cc}
\bar{A} & \bar{B} \\
\overline{A_{z}} & \overline{B_{z}}
\end{array}\right] .
$$

Hence, this and Lemma 2.9 imply that

$$
\beta e_{0}+(\alpha-\beta) e_{3}=\frac{2(2 \beta-\alpha)}{|\eta / d z|^{2}}\left[\begin{array}{cc}
A & A_{z} \\
B & B_{z}
\end{array}\right]\left[\begin{array}{cc}
\Lambda_{z \bar{z}} & \Lambda_{z} \\
\Lambda_{\bar{z}} & \Lambda
\end{array}\right]\left[\begin{array}{cc}
\bar{A} & \bar{B} \\
\overline{A_{z}} & \overline{B_{z}}
\end{array}\right] .
$$

Here we have assumed (and will continue to assume) that $\eta$ is not identically zero. This assumption means that the surface is not a horosphere.

LEMMA 3.1.

$$
\begin{aligned}
& e_{0}=g\left\{\gamma_{0}\left[\begin{array}{ll}
\Lambda & 0 \\
0 & 0
\end{array}\right]+\delta\left[\begin{array}{cc}
\Lambda_{z \bar{z}} & \Lambda_{z} \\
\Lambda_{\bar{z}} & \Lambda
\end{array}\right]\right\} g^{*}, \\
& e_{3}=g\left\{\gamma_{3}\left[\begin{array}{cc}
\Lambda & 0 \\
0 & 0
\end{array}\right]+\delta\left[\begin{array}{cc}
\Lambda_{z \bar{z}} & \Lambda_{z} \\
\Lambda_{\bar{z}} & \Lambda
\end{array}\right]\right\} g^{*},
\end{aligned}
$$

where

$$
\gamma_{0}=\frac{\beta-\alpha}{2 \beta-\alpha}, \quad \gamma_{3}=\frac{\beta}{2 \beta-\alpha}, \quad \delta=\frac{2}{|\hat{\eta}|^{2}} .
$$

LEMMA 3.2.

$$
|\eta / d z|^{2}=|\operatorname{det} g|^{2} \Lambda^{2}=4 \frac{2 \beta-\alpha}{\alpha}(\log \Lambda)_{z \bar{z}}
$$

PROOF. By Lemma 3.1,

$$
\begin{aligned}
-1 & =\left\langle e_{0}, e_{0}\right\rangle_{L}=-\operatorname{det} e_{0}=-|\operatorname{det} g|^{2}\left\{\gamma_{0} \delta \Lambda^{2}+\delta^{2}\left(\Lambda_{z \bar{z}} \Lambda-\Lambda_{z} \Lambda_{\bar{z}}\right)\right\} \\
1 & =\left\langle e_{3}, e_{3}\right\rangle_{L}=-\operatorname{det} e_{3}=-|\operatorname{det} g|^{2}\left\{-\gamma_{3} \delta \Lambda^{2}+\delta^{2}\left(\Lambda_{z \bar{z}} \Lambda-\Lambda_{z} \Lambda_{\bar{z}}\right)\right\} .
\end{aligned}
$$

Subtracting and adding these, we have

$$
\begin{gathered}
-2=-|\operatorname{det} g|^{2}\left(\gamma_{0}+\gamma_{3}\right) \delta \Lambda^{2}=-|\operatorname{det} g|^{2} \delta \Lambda^{2}, \text { i.e., }|\eta / d z|^{2}=|\operatorname{det} g|^{2} \Lambda^{2} \\
0=-|\operatorname{det} g|^{2}\left\{\left(\gamma_{0}-\gamma_{3}\right) \delta \Lambda^{2}+2 \delta^{2}\left(\Lambda_{z \bar{z}} \Lambda-\Lambda_{z} \Lambda_{\bar{z}}\right)\right\}, \\
\quad \text { i.e., } \frac{\alpha}{2 \beta-\alpha} \Lambda^{2}=2 \delta\left(\Lambda_{z \bar{z}} \Lambda-\Lambda_{z} \Lambda_{\bar{z}}\right) .
\end{gathered}
$$

By Proposition 2.8, the pseudometric $|\eta|^{2}=\left\langle d\left(e_{0}+e_{3}\right), d\left(e_{0}+e_{3}\right)\right\rangle_{L}$ has constant curvature $\alpha /(\alpha-2 \beta)(=\varepsilon)$. We set $\alpha /(\alpha-2 \beta)=\varepsilon$ and note that $\alpha: \beta=2 \varepsilon:(\varepsilon-1)$. It 
follows from the Frobenius theorem that there exists a holomorphic map $h$ from the universal cover $\tilde{M}$ to the Riemann sphere $\mathbf{S}^{2}$ or the complex plane $\mathbf{C}$ or the Poincaré disk $\mathbf{D}$ such that the pull-back of the standard metric via $h$ coincides with $|\eta|^{2}$, that is,

$$
1+\varepsilon|h|^{2}>0, \quad|\eta|^{2}=\frac{4|d h|^{2}}{\left(1+\varepsilon|h|^{2}\right)^{2}} .
$$

We call this $h$ the developing map.

Exchanging with $h$, we reexamine (3.2) and (3.3). Since the pair $A, B$ has the ambiguity of multiplication by non-zero holomorphic functions, we can start with the assumption

$$
(\operatorname{det} g=) A B_{z}-A_{z} B=h_{z} .
$$

It follows from Lemma 3.2 and (3.4) that

$$
\Lambda=\frac{2}{1+\varepsilon|h|^{2}} .
$$

It is straightforward to calculate that

$$
\begin{aligned}
\Lambda_{z} & =-2 \varepsilon \frac{h_{z} \bar{h}}{\left(1+\varepsilon|h|^{2}\right)^{2}}, \\
\Lambda_{z \bar{z}} & =-2 \varepsilon\left|h_{z}\right|^{2} \frac{\left(1-\varepsilon|h|^{2}\right)}{\left(1+\varepsilon|h|^{2}\right)^{3}} .
\end{aligned}
$$

Substituting (3.4), (3.6), (3.7), (3.8) into (3.2), (3.3), respectively, we have

$$
\begin{aligned}
e_{0} & =\left[\begin{array}{ll}
A & A_{z} \\
B & B_{z}
\end{array}\right]\left\{\gamma_{0}\left[\begin{array}{cc}
\lambda & 0 \\
0 & 0
\end{array}\right]+\left[\begin{array}{cc}
-\varepsilon \frac{1-\varepsilon|h|^{2}}{1+\varepsilon|h|^{2}} & -\varepsilon \frac{\bar{h}}{h_{z}} \\
-\varepsilon \frac{h}{h_{z}} & \frac{1+\varepsilon|h|^{2}}{\left|h_{z}\right|^{2}}
\end{array}\right]\right\}\left[\begin{array}{cc}
\bar{A} & \bar{B} \\
\overline{A_{z}} & \overline{B_{z}}
\end{array}\right] \\
& =\left[\begin{array}{ll}
A & A_{z} / h_{z} \\
B & B_{z} / h_{z}
\end{array}\right]\left\{\gamma_{0}\left[\begin{array}{ll}
\lambda & 0 \\
0 & 0
\end{array}\right]+\left[\begin{array}{cc}
-\varepsilon \frac{1-\varepsilon|h|^{2}}{1+\varepsilon|h|^{2}} & -\varepsilon \bar{h} \\
-\varepsilon h & 1+\varepsilon|h|^{2}
\end{array}\right]\right\}\left[\begin{array}{cc}
\bar{A} & \bar{B} \\
\overline{A_{z}} / \overline{h_{z}} & \overline{B_{z}} / \overline{h_{z}}
\end{array}\right] \\
& =\left[\begin{array}{cc}
A & A_{z} / h_{z} \\
B & B_{z} / h_{z}
\end{array}\right]\left[\begin{array}{cc}
\frac{1+\varepsilon^{2}|h|^{2}}{1+\varepsilon|h|^{2}} & -\varepsilon \bar{h} \\
-\varepsilon h & 1+\varepsilon|h|^{2}
\end{array}\right]\left[\begin{array}{cc}
\bar{A} & \bar{B} \\
\overline{A_{z}} / \overline{h_{z}} & \overline{B_{z}} / \overline{h_{z}}
\end{array}\right],
\end{aligned}
$$

and

$$
\begin{aligned}
e_{3} & =\left[\begin{array}{ll}
A & A_{z} / h_{z} \\
B & B_{z} / h_{z}
\end{array}\right]\left\{\gamma_{3}\left[\begin{array}{cc}
\lambda & 0 \\
0 & 0
\end{array}\right]-\left[\begin{array}{cc}
-\varepsilon \frac{1-\varepsilon|h|^{2}}{1+\left.\varepsilon h\right|^{2}} & -\varepsilon \bar{h} \\
-\varepsilon h & 1+\varepsilon|h|^{2}
\end{array}\right]\right\}\left[\begin{array}{cc}
\bar{A} & \bar{B} \\
\overline{A_{z}} / \overline{h_{z}} & \overline{B_{z}} / \overline{h_{z}}
\end{array}\right] \\
& =\left[\begin{array}{cc}
A & A_{z} / h_{z} \\
B & B_{z} / h_{z}
\end{array}\right]\left[\begin{array}{cc}
\frac{1-\varepsilon^{2}|h|^{2}}{1+\varepsilon|h|^{2}} & \varepsilon \bar{h} \\
\varepsilon h & -\left(1+\varepsilon|h|^{2}\right)
\end{array}\right]\left[\begin{array}{cc}
\bar{A} & \bar{B} \\
\overline{A_{z}} / \overline{h_{z}} & \overline{B_{z}} / \overline{h_{z}}
\end{array}\right] .
\end{aligned}
$$


Introducing the three matrices $\mathcal{G}=\left[\begin{array}{cc}A & A_{z} / h_{z} \\ B & B_{z} / h_{z}\end{array}\right]$,

$$
\mathcal{H}=\left[\begin{array}{cc}
\frac{1+\varepsilon^{2}|h|^{2}}{1+\varepsilon|h|^{2}} & -\varepsilon \bar{h} \\
-\varepsilon h & 1+\varepsilon|h|^{2}
\end{array}\right] \text { and } \tilde{\mathcal{H}}=\left[\begin{array}{cc}
\frac{1-\varepsilon^{2}|h|^{2}}{1+\varepsilon|h|^{2}} & \varepsilon \bar{h} \\
\varepsilon h & -\left(1+\varepsilon|h|^{2}\right)
\end{array}\right] \text {, }
$$

we have

$$
e_{0}=\mathcal{G} \mathcal{H} \mathcal{G}^{*}, \quad e_{3}=\mathcal{G} \tilde{\mathcal{H}} \mathcal{G}^{*} .
$$

By straightforward calculation using (3.5), i.e., $A_{z} B-A B_{z}=h_{z}$, we have

$$
\mathcal{G}^{-1} d \mathcal{G}=\left[\begin{array}{cc}
0 & \theta \\
d h & 0
\end{array}\right], \quad \text { where } \theta=\frac{B_{z} A_{z z}-A_{z} B_{z z}}{\left(h_{z}\right)^{2}} d z .
$$

The one-form $\theta$ is also written as

$$
\theta=\frac{1}{A} d\left(\frac{d A}{d h}\right)=\frac{1}{B} d\left(\frac{d B}{d h}\right) .
$$

Note that $\theta$ is a one-form defined on $\tilde{M}$.

In the following, we describe the fundamental forms in terms of $h$ and $\theta$. It is not difficult to calculate that

$$
d e_{0}=\mathcal{G}\left(\left[\begin{array}{cc}
\star & \left(1+\varepsilon|h|^{2}\right) \theta+\frac{1-\varepsilon}{1+\varepsilon|h|^{2}} d \bar{h} \\
\frac{1-\varepsilon}{1+\varepsilon|h|^{2}} d h+\left(1+\varepsilon|h|^{2}\right) \bar{\theta} & 0
\end{array}\right]\right) \mathcal{G}^{*} .
$$

Hence, we have

$$
\begin{aligned}
\mathrm{I}=-\operatorname{det}\left(d e_{0}\right) & =\left|\frac{1-\varepsilon}{1+\varepsilon|h|^{2}} d h+\left(1+\varepsilon|h|^{2}\right) \bar{\theta}\right|^{2} \\
& =\frac{(1-\varepsilon)^{2}}{\left(1+\varepsilon|h|^{2}\right)^{2}}|d h|^{2}+(1-\varepsilon) \theta d h+(1-\varepsilon) \bar{\theta} d \bar{h}+\left(1+\varepsilon|h|^{2}\right)^{2}|\theta|^{2} .
\end{aligned}
$$

As a by-product of this formula, we obtain the following lemma:

LEMMA 3.3. $\theta$ dh and $\left(1+\varepsilon|h|^{2}\right)^{2}|\theta|^{2}$ are well-defined on $M$.

It is not difficult to calculate that

$$
d e_{3}=\mathcal{G}\left[\begin{array}{cc}
\star & -\left(1+\varepsilon|h|^{2}\right) \theta+\frac{1+\varepsilon}{1+\varepsilon|h|^{2}} d \bar{h} \\
\frac{1+\varepsilon}{1+\varepsilon|h|^{2}} d h-\left(1+\varepsilon|h|^{2}\right) \bar{\theta} & 0
\end{array}\right] \mathcal{G}^{*} .
$$

Hence, we have

$$
\mathrm{III}=-\operatorname{det}\left(d e_{3}\right)=\left|\frac{1+\varepsilon}{1+\varepsilon|h|^{2}} d h-\left(1+\varepsilon|h|^{2}\right) \bar{\theta}\right|^{2}
$$


190

$$
=\left(\frac{1+\varepsilon}{1+\varepsilon|h|^{2}}\right)^{2}|d h|^{2}-(1+\varepsilon) \theta d h-(1+\varepsilon) \bar{\theta} d \bar{h}+\left(1+\varepsilon|h|^{2}\right)^{2}|\theta|^{2} .
$$

It follows that

$$
\begin{aligned}
\alpha \mathrm{I}-2 \beta \mathrm{I} & =\alpha\left|d e_{0}\right|_{L}^{2}+\beta\left\{\left|d e_{0}+d e_{3}\right|_{L}^{2}-\left|d e_{0}\right|_{L}^{2}-\left|d e_{3}\right|_{L}^{2}\right\} \\
& =(\alpha-\beta)\left|d e_{0}\right|_{L}^{2}+\beta\left|d e_{0}+d e_{3}\right|_{L}^{2}-\beta\left|d e_{3}\right|_{L}^{2} \\
& =(\alpha-2 \beta)\left\{\left(1+\varepsilon|h|^{2}\right)^{2}|\theta|^{2}-\frac{(1-\varepsilon)^{2}|d h|^{2}}{\left(1+\varepsilon|h|^{2}\right)^{2}}\right\} .
\end{aligned}
$$

From the argument above, one can understand the following:

The GÁlveZ-MARTíneZ-MilÁn FORMUla ([5]). Let $M$ be a non-compact, simply-connected surface and $f: M \rightarrow \mathbf{H}^{3}$ a Weingarten surface satisfying $\alpha(H-1)=\beta K$, where $\alpha$ and $\beta$ are real constants with $\alpha \neq 2 \beta$. Then, there exist a meromorphic curve $\mathcal{G}: M \rightarrow \operatorname{SL}(2, \mathbf{C})$ and a pair $(h, \theta)$ consisting of a meromorphic function $h$ and $a$ holomorphic one-form $\theta$ on $M$, such that the immersion $f$ and its unit normal field $v$ can be recovered as $f=\mathcal{G} \mathcal{H} \mathcal{G}^{*}$ and $v=\mathcal{G} \tilde{\mathcal{H}} \mathcal{G}^{*}$, where

$$
\mathcal{H}=\left[\begin{array}{cc}
\frac{1+\varepsilon^{2}|h|^{2}}{1+\varepsilon|h|^{2}} & -\varepsilon \bar{h} \\
-\varepsilon h & 1+\varepsilon|h|^{2}
\end{array}\right] \text { and } \quad \tilde{\mathcal{H}}=\left[\begin{array}{cc}
\frac{1-\varepsilon^{2}|h|^{2}}{1+\varepsilon|h|^{2}} & \varepsilon \bar{h} \\
\varepsilon h & -1-\varepsilon|h|^{2}
\end{array}\right]
$$

with $\varepsilon=\alpha /(\alpha-2 \beta)$ and $1+\varepsilon|h|^{2}>0$. Moreover, the curve $\mathcal{G}$ satisfies

$$
\mathcal{G}^{-1} d \mathcal{G}=\left[\begin{array}{cc}
0 & \theta \\
d h & 0
\end{array}\right]
$$

The following formulas hold:

$$
\begin{aligned}
& \mathrm{I}=(1-\varepsilon) \theta d h+\left(\frac{(1-\varepsilon)^{2}|d h|^{2}}{\left(1+\varepsilon|h|^{2}\right)^{2}}+\left(1+\varepsilon|h|^{2}\right)^{2}|\theta|^{2}\right)+(1-\varepsilon) \bar{\theta} d \bar{h}, \\
& \alpha \mathrm{I}-2 \beta \mathrm{II}=(\alpha-2 \beta)\left(\left(1+\varepsilon|h|^{2}\right)^{2}|\theta|^{2}-\frac{(1-\varepsilon)^{2}|d h|^{2}}{\left(1+\varepsilon|h|^{2}\right)^{2}}\right)
\end{aligned}
$$

where I and II denote the first and second fundamental forms.

Conversely, let $M$ be a Riemann surface, $\mathcal{G}: M \rightarrow \operatorname{SL}(2, \mathbf{C})$ a meromorphic curve and $(h, \theta)$ a pair as above satisfying (3.14) and such that (3.16) is a positive definite metric. Then $f:=\mathcal{G} \mathcal{H G}^{*}: M \rightarrow \mathbf{H}^{3}(\mathcal{H}$ as in (3.13)), is a Weingarten surface satisfying $\alpha(H-1)=\beta K$ with $\mathrm{I}$ and $\alpha \mathrm{I}-2 \beta \mathrm{II}$ given by (3.15) and (3.16).

We finish this subsection by providing the following equation.

$$
\mathbb{I}=\frac{(\alpha-\beta) \beta}{(\alpha-2 \beta)^{2}} \frac{4|d h|^{2}}{\left(1+\varepsilon|h|^{2}\right)^{2}}-\frac{\alpha}{\alpha-2 \beta}(\theta d h+\bar{\theta} d \bar{h})+\left(1+\varepsilon|h|^{2}\right)^{2}|\theta|^{2} .
$$


3.2. Global version of the Gálvez-Martínez-Milán formula. We shall give a global version of the Gálvez-Martínez-Milán formula, and make clear what is a local invariant and what is a global invariant.

The hyperbolic Gauss map $G=A / B$ is globally-defined on $M$. We can represent $\mathcal{G}$ using $G$ as follows:

LEMMA 3.4.

$$
\mathcal{G}=\left(-G_{h}\right)^{-3 / 2}\left[\begin{array}{cc}
-G G_{h} & G G_{h h} / 2-G_{h}^{2} \\
-G_{h} & G_{h h} / 2
\end{array}\right]
$$

where $G_{h}=d G / d h, G_{h h}=d^{2} G / d h^{2}$.

PROOF. $\mathcal{G}$ is computed as

$$
\mathcal{G}=\left[\begin{array}{ll}
A & d A / d h \\
B & d B / d h
\end{array}\right]=\left[\begin{array}{cc}
G B & d(G B) / d h \\
B & d B / d h
\end{array}\right]=\frac{1}{B}\left[\begin{array}{cc}
G B^{2} & B d(G B) / d h \\
B^{2} & B d B / d h
\end{array}\right]
$$

On the other hand, substituting $A=B G$ to $A d B-B d A=d h$, we have

$$
B^{2}=-\frac{d h}{d G}=-\frac{1}{G_{h}}
$$

Eliminating $B$ from (3.18) with this, we have the assertion.

Thus, we can make a representation formula $f=\mathcal{G H} \mathcal{H}^{*}$ with $\mathcal{G}$ as in (3.17) and

$$
\mathcal{H}=\left[\begin{array}{cc}
\frac{1+\varepsilon^{2}|h|^{2}}{1+\varepsilon|h|^{2}} & -\varepsilon \bar{h} \\
-\varepsilon h & 1+\varepsilon|h|^{2}
\end{array}\right]
$$

from a meromorphic function $G$ on $M$ and a holomorphic map $h: \tilde{M} \rightarrow \mathbf{S}^{2}, \mathbf{C}$ or D. However, it is not defined on $M$ yet (merely on $\tilde{M}$, in general). We need to find the condition that $f$ is single-valued on $M$. Indeed, we prove:

Proposition 3.5. $f=\mathcal{G} \mathcal{H G}^{*}$ with $\mathcal{G}, \mathcal{H}$ as in (3.17), (3.20) is single-valued on $M$ if and only if the pseudometric $|\eta|^{2}=4|d h|^{2} /\left(1+\varepsilon|h|^{2}\right)^{2}$ is single-valued on $M$.

Proof. Suppose that $|\eta|^{2}=4|d h|^{2} /\left(1+\varepsilon|h|^{2}\right)^{2}$ is single-valued on $M$.

By (3.6) and Lemma 3.4, we have

$$
\begin{aligned}
\Lambda|A|^{2} & =\frac{2}{1+\varepsilon|h|^{2}}|G|^{2}\left|-G_{h}\right|^{-1}=\frac{2}{1+\varepsilon|h|^{2}}|G|^{2}\left|\frac{d h}{d G}\right|=2 \frac{|d h|}{1+\varepsilon|h|^{2}} \frac{|G|^{2}}{|d G|} \\
\Lambda|B|^{2} & =2 \frac{|d h|}{1+\varepsilon|h|^{2}} \frac{1}{|d G|} \\
\Lambda A \bar{B} & =2 \frac{|d h|}{1+\varepsilon|h|^{2}} \frac{G}{|d G|} .
\end{aligned}
$$


Thus, all $\Lambda|A|^{2}, \Lambda|B|^{2}, \Lambda A \bar{B}$ are single-valued on $M$. In other words, $e_{0}+e_{3}$ is single-valued on $M$ (because of (3.1)). On the other hand, $\eta \wedge \bar{\eta}$ is also single-valued on $M$. Therefore, recalling Lemma 2.9, we can conclude that $e_{0}(=f)$ is also single-valued on $M$.

Moreover, we need some lemmas.

Lemma 3.6. Let $\mathcal{G}: U \rightarrow \operatorname{SL}(2, \mathbf{C})$ be a meromorphic Legendrian map. A point $p \in U$ is a pole of $\mathcal{G}$ if and only if $p$ is a pole of $\mathcal{G}^{-1} d \mathcal{G}$.

LEMMA 3.7. For a meromorphic Legendrian map $\mathcal{G}$ as in (3.17), the one-form $\theta$ can be calculated as

$$
\theta=-\frac{1}{2}\{G ; h\} d h\left(=-\frac{1}{2}\left\{\left(\frac{G_{h h}}{G_{h}}\right)_{h}-\frac{1}{2} \frac{\left(G_{h h}\right)^{2}}{\left(G_{h}\right)^{2}}\right\} d h\right),
$$

where $\{G ; h\}$ denotes the Schwarzian derivative of $G$ with respect to $h$.

Proof. Differentiating (3.19) $B^{2}=-1 / G_{h}$ with respect to $h$, we have

$$
d B / d h=\frac{1}{2} \frac{1}{B} \frac{G_{h h}}{\left(G_{h}\right)^{2}} .
$$

Differentiating this again, we have

$$
\frac{d}{d h}\left(\frac{d B}{d h}\right)=\frac{1}{2}\left\{-\frac{1}{2} \frac{1}{B} \frac{\left(G_{h h}\right)^{2}}{\left(G_{h}\right)^{3}}+\frac{1}{B} \frac{1}{G_{h}}\left(\frac{G_{h h}}{G_{h}}\right)_{h}\right\} .
$$

Therefore, it follows from (3.10) that

$$
\theta=\frac{1}{2}\left\{-\frac{1}{2} \frac{1}{B^{2}} \frac{\left(G_{h h}\right)^{2}}{\left(G_{h}\right)^{3}}+\frac{1}{B^{2}} \frac{1}{G_{h}}\left(\frac{G_{h h}}{G_{h}}\right)_{h}\right\} d h .
$$

Again, using $B^{2}=-1 / G_{h}$, we obtain

$$
\theta=\frac{1}{2}\left\{\frac{1}{2} \frac{\left(G_{h h}\right)^{2}}{\left(G_{h}\right)^{2}}-\left(\frac{G_{h h}}{G_{h}}\right)_{h}\right\} d h=-\frac{1}{2}\{G ; h\} d h .
$$

This lemma tells us, for example, that $\alpha \mathrm{I}-2 \beta$ II is conformal to

$$
\frac{\left(1+\varepsilon|h|^{2}\right)^{2}}{4}|\{G ; h\} d h|^{2}-\frac{(1-\varepsilon)^{2}|d h|^{2}}{\left(1+\varepsilon|h|^{2}\right)^{2}} .
$$

Therefore we have:

THEOREM 3.8. Let $G$ be a meromorphic function on a Riemann surface M. Let $|\eta|^{2}$ be a Hermitian pseudometric on $M$ of constant curvature $\varepsilon$, and $h$ the developing map. Suppose that the quadratic differential form (3.21) is definite on $M$. Then $f:=\mathcal{G H G}^{*}$, determined by (3.17) and (3.20), is a Weingarten immersion from $M$ to $\mathbf{H}^{3}$ with $2 \varepsilon(H-1)=$ $(\varepsilon-1) K$. 
Conversely, any Weingarten surface of $\alpha(H-1)=\beta K(\alpha \neq 2 \beta)$, except a horosphere, has this representation in terms of $(G, h)$.

REMARK. (1) It has been already proved in [7] that the solution to the differential equation (3.14) is described as (3.17).

(2) Under the condition $h(z)=z$, the formula $f:=\mathcal{G H G}^{*}$ with (3.17) and (3.20), was already seen in [5, Theorem 4], where the condition $h(z)=z$ is caused by their assumption that $M$ is simply-connected and complete. In contrast, Theorem 3.8 is devoted to surfaces of non-trivial topology. The period condition is clarified, indeed, it is that $|\eta|^{2}$ is single-valued on $M$.

\section{Surfaces with constant harmonic-mean curvature one}

The following proposition follows easily from (2.15) and Proposition 2.2, respectively.

Proposition 4.1. If $f: M \rightarrow \mathbf{H}^{3}$ is a Weingarten surface satisfying $H-1=\lambda K$ $(\lambda \neq 1 / 2)$, then the Gaussian curvature $K$ satisfies the following inequalities:

(i) If $\lambda=0$, then $K \leq 0$.

(ii) If $\lambda<1 / 2(\neq 0)$, then $K \leq 0$ or $K \geq(1-2 \lambda) / \lambda^{2}$.

(iii) If $\lambda>1 / 2$, then $K \leq(1-2 \lambda) / \lambda^{2}$ or $K \geq 0$.

The mean curvature $H$ satisfies the following:

(i) If $0<\lambda<1 / 2$, then $H \leq 1$ or $H \geq(1-\lambda) / \lambda$.

(ii) If $\lambda<0$ or $\lambda>1 / 2$, then $H \leq(1-\lambda) / \lambda$ or $H \geq 1$.

4.1. Basics. In this section, we study the case $\lambda=1$ for Weingarten surfaces satisfying $H-1=\lambda K$, that is, the case $H-1=K$. As stated in the previous section, a Weingarten surface satisfying $H-1=K$ has constant harmonic-mean curvature one, and we call it an HMC-1 surface.

By Lemma 2.1 and (2.16), an HMC-1 surface satisfies

$$
\begin{aligned}
\left\langle d e_{0}+d e_{3}, d e_{0}+d e_{3}\right\rangle_{L} & =-K(\mathrm{I}-2 \mathbb{I}), \\
(\mathbb{I I I}=)\left\langle d e_{3}, d e_{3}\right\rangle_{L} & =-H(\mathrm{I}-2 \mathrm{II}) .
\end{aligned}
$$

These two quadratic differentials are conformally equivalent. The formulas (4.1) and (4.2) become

$$
\begin{aligned}
& |\eta|^{2}\left(=\left(\omega^{1}-\omega_{1}^{3}\right)^{2}+\left(\omega^{2}-\omega_{2}^{3}\right)^{2}\right)=-K(\mathrm{I}-2 \mathrm{II}), \\
& |\pi|^{2}\left(=\left(\omega_{1}^{3}\right)^{2}+\left(\omega_{2}^{3}\right)^{2}\right)=-H(\mathrm{I}-2 \mathrm{I}) .
\end{aligned}
$$

Consequently, we obtain the following lemma:

LEMMA 4.2. $\eta(p)=0$ if and only if $p$ is an umbilical point with $\mathbb{I}_{p}=\mathrm{I}_{p}$, i.e., $a$ point where $K=0$ and $H=1 . \pi(p)=0$ if and only if $p$ is a totally geodesic point, i.e., a point where $K=-1$ and $H=0$. 
LEMmA 4.3. The Gaussian and mean curvatures $K, H$ are given by

$$
K=\frac{|\eta|^{2}}{|\pi|^{2}-|\eta|^{2}}, \quad H=\frac{|\pi|^{2}}{|\pi|^{2}-|\eta|^{2}} .
$$

Proof. It follows from (4.3) and (4.4) that $H|\eta|^{2}=K|\pi|^{2}$. This formula implies the assertion, since $H-1=K$.

Note that

$$
K \leq-1(H \leq 0) \text { or } K \geq 0(H \geq 1)
$$

for HMC-1 surfaces, because of Proposition 4.1. It follows from Lemma 4.3 that $K \leq-1$ if and only if $|\pi|^{2}<|\eta|^{2}$, and that $K \geq 0$ if and only if $|\pi|^{2}>|\eta|^{2}$.

LEMMA 4.4.

$$
\bar{q} \eta=-K \bar{\pi} .
$$

Proof. By (2.14), we have $|q|^{2}=H^{2}-(K+1)=H^{2}-H=H(H-1)=H K$. Moreover, using (2.12), we have $\eta=\bar{\omega}-\pi=\bar{\omega}-(q \omega+H \bar{\omega})=-q \omega+(1-H) \bar{\omega}=-q \omega-$ $K \bar{\omega}$, therefore, $\bar{q} \eta=-|q|^{2} \omega-K \bar{q} \bar{\omega}=-H K \omega-K \bar{q} \bar{\omega}=(-K)(H \omega+\bar{q} \bar{\omega})=(-K) \bar{\pi}$.

LEMMA 4.5. Let $\mathrm{I}^{2,0}$ and $\mathbb{I}^{2,0}$ denote the $(2,0)$-parts of the complexification of the fundamental forms I and II, respectively. Then

$$
\mathrm{I}^{2,0}=2 \mathrm{I}^{2,0}=\eta \bar{\pi},
$$

and $\eta \bar{\pi}$ is a holomorphic quadratic differential on $M$.

PROOF. (4.6) is obtained by rewriting I and II with $\eta=\bar{\omega}-\pi$. Indeed,

$$
\mathrm{I}(=\omega \bar{\omega})=\eta \bar{\pi}+|\eta|^{2}+|\pi|^{2}+\bar{\eta} \pi, \quad \mathrm{II}(=\operatorname{Re}(\omega \pi))=\frac{1}{2}\left(\eta \bar{\pi}+2|\pi|^{2}+\bar{\eta} \pi\right) .
$$

It follows from the formulas in (2.9), (2.10) that $d \bar{\pi}=\sqrt{-1} \omega_{2}^{1} \wedge \bar{\pi}$ and $d \eta=-\sqrt{-1} \omega_{2}^{1} \wedge \eta$. They imply that $\eta \bar{\pi}$ is holomorphic.

Proposition 4.6. The pseudometric $|\pi|^{2}(=\mathrm{III})$ has the Gaussian curvature $K /(K+1)\left(=|\eta|^{2} /|\pi|^{2}\right)$.

PROOF. Since $d \bar{\pi}=\sqrt{-1} \omega_{2}^{1} \wedge \bar{\pi}$, we may regard $\omega_{2}^{1}$ as the connection form for $|\pi|^{2}$. Moreover, its exterior differential is computed as

$$
\begin{aligned}
d \omega_{2}^{1} & =-\frac{\sqrt{-1}}{2}(\pi \wedge \bar{\pi}+\omega \wedge \bar{\omega})=-\frac{\sqrt{-1}}{2}\left(\pi \wedge \bar{\pi}+\frac{1}{K+1} \bar{\pi} \wedge \pi\right) \\
& =-\frac{\sqrt{-1}}{2}\left(\frac{-K}{K+1} \bar{\pi} \wedge \pi\right)=\frac{K}{K+1} \frac{\sqrt{-1}}{2} \bar{\pi} \wedge \pi,
\end{aligned}
$$

because of (2.10) and (2.11), which proves the assertion. 
LEMMA 4.7.

$$
\bar{\partial} \partial\left(e_{0}+e_{3}\right)=\frac{1}{2} e_{0} \otimes(\bar{\eta} \wedge \eta)
$$

Proof. Because $d\left(e_{0}+e_{3}\right)=e \bar{\eta}+\bar{e} \eta$, we have $\partial\left(e_{0}+e_{3}\right)=\bar{e} \eta$. Furthermore, taking $\bar{\partial}$, we can calculate as follows:

$$
\begin{aligned}
\bar{\partial} \partial\left(e_{0}+e_{3}\right) & =\bar{\partial} \bar{e} \wedge \eta+\bar{e} \otimes \bar{\partial} \eta \\
& =d \bar{e} \wedge \eta+\bar{e} \otimes d \eta \quad \text { (since } \eta \text { is a }(1,0) \text {-form, }) \\
& =\left(e_{0} \otimes \frac{1}{2} \omega+\sqrt{-1} \bar{e} \otimes \omega_{2}^{1}+e_{3} \otimes \frac{1}{2} \bar{\pi}\right) \wedge \eta+\bar{e} \otimes\left(-\sqrt{-1} \omega_{2}^{1} \wedge \eta\right) \\
& =\frac{1}{2} e_{0} \otimes(\omega \wedge \eta) \quad(\text { since } \bar{\pi} \text { is a }(1,0) \text {-form, }) \\
& =\frac{1}{2} e_{0} \otimes((\omega-\bar{\pi}) \wedge \eta) \quad(\text { since } \bar{\pi} \text { is a }(1,0) \text {-form, }) \\
& =\frac{1}{2} e_{0} \otimes(\bar{\eta} \wedge \eta) .
\end{aligned}
$$

By (3.11) and (3.12), we have

$$
\mathbb{I}=\theta d h+\left(1-|h|^{2}\right)^{2}|\theta|^{2}+\bar{\theta} d \bar{h} .
$$

In particular,

$$
|\pi|^{2}=\mathbb{I}^{1,1}=\left(1-|h|^{2}\right)^{2}|\theta|^{2} .
$$

It follows from Lemma 4.3, (3.4) and (4.7) that

$$
K=\frac{4|d h|^{2}}{\left(1-|h|^{2}\right)^{4}|\theta|^{2}-4|d h|^{2}}, \quad H=\frac{\left(1-|h|^{2}\right)^{4}|\theta|^{2}}{\left(1-|h|^{2}\right)^{4}|\theta|^{2}-4|d h|^{2}} .
$$

\section{Fronts with constant harmonic-mean curvature one}

5.1. Definition. Let $M$ be a Riemann surface. Given a meromorphic function $G: M \rightarrow \mathbf{C} \cup\{\infty\}$ and a pseudometric $|\eta|^{2}$ of constant curvature -1 on $M$, we can define a map $f=\mathcal{G} \mathcal{H} \mathcal{G}^{*}: M \rightarrow \mathbf{H}^{3}$ using (3.4), (3.17) and (3.20). Since $\mathcal{G}$ has poles $\left\{p_{i}\right\}$ in general, $f$ should be considered a map on $M \backslash\left\{p_{i}\right\}$. However, in such a case, we redefine $M$ to be $M \backslash\left\{p_{i}\right\}$.

We call $f$ an $H M C$ - 1 map associated with $\left(G,|\eta|^{2}\right)$. By definition, the regular image of an HMC-1 map forms an immersed surface with constant harmonic-mean curvature one whose unit normal vector field is $v=\mathcal{G}\left[\begin{array}{cc}1 & -\bar{h} \\ -h & -1+|h|^{2}\end{array}\right] \mathcal{G}^{*}$. Though $f$ may fail to be an immersion, the unit normal $v$ is defined across the singularities. Hence, the following definition does make sense. 
An HMC-1 map $f$ is called an HMC-1 front if $(f, v): M \rightarrow T_{1} \mathbf{H}^{3}\left(\cong T_{1}^{*} \mathbf{H}^{3}\right)$ is an immersion, where $T_{1} \mathbf{H}^{3}\left(T_{1}^{*} \mathbf{H}^{3}\right)$ denotes the unit (co)tangent bundle over $\mathbf{H}^{3}$. (The term front comes from wave fronts in the theory of singularities.) It is obvious from the definition that the formulas for HMC-1 surfaces in the previous section can be applied for HMC-1 fronts.

Proposition 5.1. For an HMC-1 map $f: M \rightarrow \mathbf{H}^{3}$, the following three conditions are equivalent:

(1) $f$ is an HMC-1 front.

(2) The (1, 1)-part $\mathrm{I}^{1,1}\left(=|\eta|^{2}+|\pi|^{2}\right)$ of the first fundamental form $\mathrm{I}=|\eta+\pi|^{2}$ is a Riemannian metric on $M$.

(3) $\mathcal{G}: \tilde{M} \rightarrow \operatorname{SL}(2$, C) is non-singular.

PROOF. We can put (1)-(3) in different words as follows:

(1) $|d f|^{2}$ and $|d \nu|^{2}$ never vanish simultaneously, that is, $\left|2 d h /\left(1-|h|^{2}\right)+\left(1-|h|^{2}\right) \bar{\theta}\right|^{2}$ and $\left(1-|h|^{2}\right)^{2}|\theta|^{2}$ never vanish simultaneously.

(2) $\mathrm{I}^{1,1}=4|d h|^{2} /\left(1-|h|^{2}\right)^{2}+\left(1-|h|^{2}\right)^{2}|\theta|^{2}$ never vanishes.

(3) Either $\theta \neq 0$, or both $\theta=0$ and $d h \neq 0$.

Then, it is not difficult to see the equivalency.

REMARK. $\quad T_{1} \mathbf{H}^{3}\left(\cong T_{1}^{*} \mathbf{H}^{3}\right)$ has a canonical Riemannian metric, which is called the Sasakian metric. We denote by $\mathrm{I}^{S}$ the pull-back of the Sasakian metric via the map $(f, v)$. $\mathrm{I}^{S}$ is a Riemannian metric on $M$ for a front $f: M \rightarrow \mathbf{H}^{3}$. Indeed, $\mathrm{I}^{S}=|d f|^{2}+|d \nu|^{2}=$ $|\eta+\pi|^{2}+|\pi|^{2}$. $\mathrm{I}^{S}$ is not conformally equivalent to $\mathrm{I}^{1,1}$ in general.

It is clear from Proposition 5.1 that a singularity of an HMC-1 front is a point where

$$
(\eta+\pi) \wedge(\bar{\eta}+\bar{\pi})=0 \Longleftrightarrow \eta \wedge \bar{\eta}+\pi \wedge \bar{\pi}=0 \Longleftrightarrow|\eta|^{2}=|\pi|^{2}
$$

Proposition 5.2. There are no compact HMC-1 fronts.

Proof. Suppose, by way of contradiction, that there exists a compact HMC-1 front $f: M \rightarrow \mathbf{H}^{3}$.

It follows from Lemma 4.7 that

$$
\left(e_{0}+e_{3}\right)_{z \bar{z}}=\frac{|\eta / d z|^{2}}{2} e_{0}
$$

Taking the trace of both side, we have

$$
\left(\operatorname{tr}\left(e_{0}+e_{3}\right)\right)_{z \bar{z}}=\frac{|\eta / d z|^{2}}{2} \operatorname{tr} e_{0}=|\eta / d z|^{2} x_{0} \geq 0 .
$$

Hence, $\operatorname{tr}\left(e_{0}+e_{3}\right)$ is a subharmonic function on $M$. It must be constant, since $M$ is compact. Again by (5.1), we have $\eta=0$, a contradiction. 
5.2. Weak completeness. We say that an HMC-1 front $f: M \rightarrow \mathbf{H}^{3}$ is weakly complete if $\mathrm{I}^{1,1}$, the $(1,1)$-part the first fundamental form $\mathrm{I}$, is a complete Riemannian metric on $M$ (cf. [6]).

PROPOSITION 5.3. For an HMC-1 front, weak completeness is equivalent to the completeness of $\mathrm{I}^{S}$, the induced metric of the Sasakian metric.

Proof. Let $\gamma:[0, \infty) \rightarrow M$ be an arbitrary divergent path. Recall that $\mathrm{I}^{S}=\mid \eta+$ $\left.\pi\right|^{2}+|\pi|^{2}$ and $I^{1,1}=|\eta|^{2}+|\pi|^{2}$. We wish to prove that, if $\gamma$ has infinite length with respect to one of the two metrics, then it also has infinite length with respect to the other metric. When $\int_{\gamma}|\pi|=\infty$, it is trivial that $\gamma$ has infinite length with respect to both metrics $\mathrm{I}^{S}$ and $\mathrm{I}^{1,1}$. Hence, we have only to give a proof under the assumption $\int_{\gamma}|\pi|<\infty$.

(a) Suppose that $\mathrm{I}^{1,1}$ is complete. Clearly, $\int_{\gamma}|\eta|=\infty$. If we divide the interval $[0, \infty)$ so that

$$
[0, \infty)=J_{+} \cup J_{-}, \text {where } J_{+}=\{|\eta| \geq|\pi|\}, J_{-}=\{|\eta|<|\pi|\},
$$

then $\int_{J_{+}}|\eta|=\infty$, because $\int_{J_{-}}|\eta|<\int_{J_{-}}|\pi|<\infty$. Thus,

$$
\begin{aligned}
& \int_{\gamma} \sqrt{|\eta+\pi|^{2}+|\pi|^{2}} \geq \int_{\gamma}|\eta+\pi| \geq \int_{\gamma}|| \eta|-| \pi|| \\
\geq & \int_{J_{+}}|\eta|-|\pi|=\int_{J_{+}}|\eta|-\int_{J_{+}}|\pi|=\infty-(\text { finite value })=\infty .
\end{aligned}
$$

Therefore, $\mathrm{I}^{S}$ is complete.

(b) Conversely, we suppose $\mathrm{I}^{S}$ is complete. Clearly, $\int_{\gamma}|\eta+\pi|=\infty$. If we divide the interval $[0, \infty)$ so that

$$
[0, \infty)=J_{+}^{\prime} \cup J_{-}^{\prime}, \text { where } J_{+}^{\prime}=\{|\eta+\pi| \geq|\pi|\}, J_{-}^{\prime}=\{|\eta+\pi|<|\pi|\},
$$

then $\int_{J_{+}^{\prime}}|\eta+\pi|=\infty$, because $\int_{J_{-}^{\prime}}|\eta+\pi|<\int_{J_{-}^{\prime}}|\pi|=\infty$. Thus

$$
\begin{aligned}
& \int_{\gamma} \sqrt{|\eta|^{2}+|\pi|^{2}} \geq \int_{\gamma}|\eta| \geq \int_{\gamma}|| \eta+\pi|-|-\pi|| \\
\geq & \int_{J_{+}^{\prime}}|\eta+\pi|-|\pi| \geq \int_{J_{+}^{\prime}}|\eta+\pi|-\int_{J_{+}^{\prime}}|\pi|=\infty .
\end{aligned}
$$

Therefore $\mathrm{I}^{1,1}$ is complete.

Note that an HMC-1 front is weakly complete if it is complete (in the usual sense), because $\mathrm{I}^{S}=|d f|^{2}+|d \nu|^{2}$ is complete if $\mathrm{I}=|d f|^{2}$ is complete.

5.3. HMC-1 fronts of finite topology. There are two kind of ends for (weakly) complete HMC-1 fronts of finite topology. One is conformally equivalent to the punctured disk $\Delta^{*}=\{z ; 0<|z|<1\}$, and the other is conformally equivalent to the annulus 
$A_{r}=\{z ; r<|z|<1\}$. We shall call the former a puncture-type end, the latter an annular end. For a puncture-type end $\Delta^{*}$, we also call a point $z=0$ an end. For an annular end $A_{r}$, we also call the boundary $|z|=r$ an end.

5.4. Examples. We show some examples of (weakly) complete HMC-1 fronts of finite topology.

In this section, we denote by $d s_{H}^{2}$ the Poincaré metric on the unit disk, and in the figures, the hyperbolic three-space $\mathbf{H}^{3}$ is realized by the Poincaré ball model.

EXAMPLE 1. For a positive number $\alpha$, consider

$$
G(z)=z,|\eta|^{2}=\frac{4|\alpha|^{2}|z|^{2 \alpha-2}}{\left(1-|z|^{2 \alpha}\right)^{2}}|d z|^{2}\left(=h^{*} d s_{H}^{2} \text { where } h(z)=z^{\alpha}\right)
$$

on

$$
M= \begin{cases}\Delta=\{z ;|z|<1\} & \text { if } \alpha=1, \\ \Delta \backslash\{0\} & \text { otherwise. }\end{cases}
$$

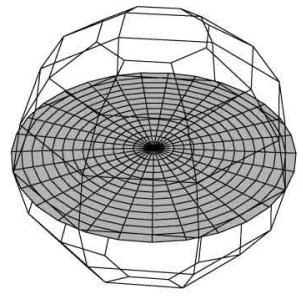

$(G, h)=(z, z)$

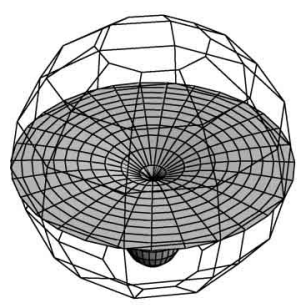

$(G, h)=(z, \sqrt{z})$

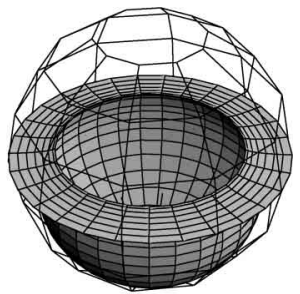

$(G, h)=\left(z, z^{2}\right)$

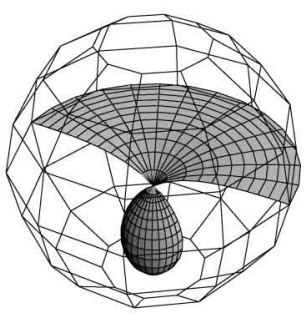

$(G, h)=(z, \sqrt{z})$ (half cut)

FIGURE 1 


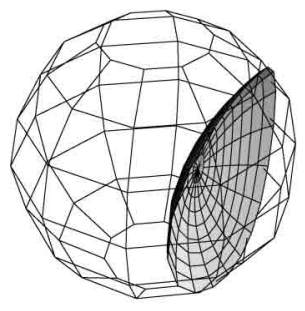

$(G, h)=(\exp z, z)$

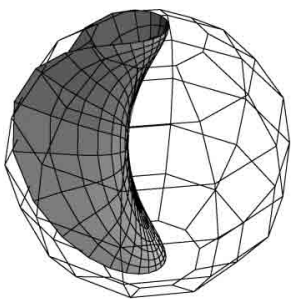

$(G, h)=(\exp 2 \sqrt{2} z, z)$

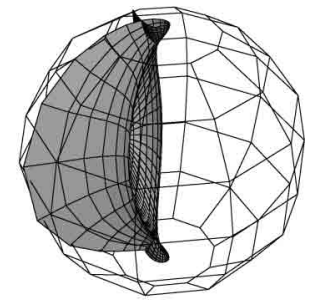

$(G, h)=(\exp 4 z, z)$

FIGURE 2

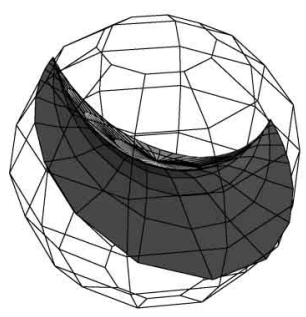

$(G, h)=(z+1 / z, z)$

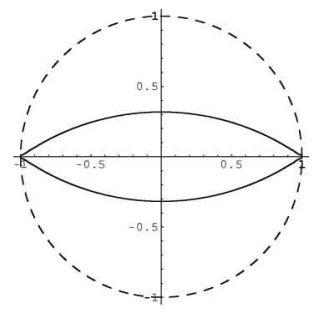

singular locus in $\Delta$

FIGURE 3

Then the HMC-1 front $f: M \rightarrow \mathbf{H}^{3}$ associated with $\left(G,|\eta|^{2}\right)$ satisfies

$$
\begin{aligned}
& \theta=\frac{1-\alpha^{2}}{4 \alpha} z^{-\alpha-1} d z, d h=\alpha z^{\alpha-1} d z, Q(=\eta \bar{\pi})=\frac{1-\alpha^{2}}{2 z^{2}} d z^{2}, \\
& |\pi|^{2}=\frac{\left|\alpha^{2}-1\right|^{2}}{16|\alpha|^{2}} \frac{\left(1-|z|^{2 \alpha}\right)^{2}}{|z|^{2 \alpha+2}}|d z|^{2} .
\end{aligned}
$$

If $\alpha=1$, then $f$ is complete and totally geodesic. If $\alpha \neq 1$, then $f$ is weakly complete and has a puncture-type end at $z=0$ and an annular end at $|z|=1$. Its singular locus is the circle

$$
|z|=\left(-\sqrt{\frac{2 \alpha^{2}}{\alpha^{2}-1}}+\sqrt{\frac{2 \alpha^{2}}{\alpha^{2}-1}+1}\right)^{1 / \alpha} .
$$

$K \geq 0$ inside this circle, and $K \leq-1$ outside the circle. 
EXAMPLE 2. For a non-zero complex number $k$, consider $G(z)=\exp (k z),|\eta|^{2}=$ $d s_{H}^{2}$ on $\Delta$, then the associated HMC-1 front satisfies

$$
\theta=\frac{k^{2}}{4} d z, d h=d z, Q=\frac{k^{2}}{2} d z^{2},|\pi|^{2}=\frac{|k|^{4}}{16}\left(1-|z|^{2}\right)^{2}|d z|^{2} .
$$

Its singular locus is $|z|^{2}=1-2 \sqrt{2} /|k|$. In particular, it has no singularities if $|k|<2 \sqrt{2}$.

EXAMPLE 3. Consider $G(z)=z+1 / z,|\eta|^{2}=d s_{H}^{2}$ on $\Delta$. Then the associated HMC-1 front satisfies

$$
\theta=\frac{3}{\left(z^{2}-1\right)^{2}} d z, d h=d z, Q=\frac{6}{\left(z^{2}-1\right)^{2}} d z^{2},|\pi|^{2}=\frac{9\left(1-|z|^{2}\right)^{2}}{\left|z^{2}-1\right|^{4}}|d z|^{2} .
$$

Its singular locus is

$$
C: 2\left|z^{2}-1\right|^{2}=3\left(1-|z|^{2}\right)^{2} .
$$

$f$ has an annular end at $|z|=1$, and the singular locus $C$ accumulates at $z= \pm 1$.

\section{References}

[ 1] R. BRYANT, Surfaces of mean curvature one in hyperbolic space, in Théorie des variétés minimales et applications, Astérisque 154-155 (1988), 321-347.

[2] P. Collin, L. Hauswirth and H. Rosenberg, The geometry of finite topology Bryant surfaces, Ann. of Math. (2) 153 (2001), no. 3, 623-659.

[ 3 ] C. L. EPSTEIN, Envelopes of Horospheres and Weingarten Surfaces in Hyperbolic 3-Space, unpublished.

[4] J. A. GÁlvez, A. Martínez and F. Milán, Flat surfaces in hyperbolic 3-space, Math. Ann., 316 (2000), no. 3, 419-435.

[ 5 ] J. A. Gálvez, A. Martínez and F. Milán, Complete linear Weingarten surfaces of Bryant type. A Plateau problem at infinity, Trans. Amer. Math. Soc. 356 (2004), no. 9, 3405-3428.

[6] M. Kokubu, W. Rossman, K. Saji, M. Umehara and K. Yamada, Singularities of flat fronts in hyperbolic space, Pacific J. Math. 221 (2005), no. 2, 303-351.

[7] M. Kokubu, M. Umehara and K. Yamada, An elementary proof of Small's formula for null curves in $\operatorname{PSL}(2, \mathbf{C})$ and an analogue for Legendrian curves in PSL(2, C), Osaka J. Math. 40 (2003), no. 3, 697-715.

[ 8 ] M. Kokubu, M. Umehara and K. Yamada, Flat fronts in hyperbolic 3-space, Pacific J. Math. 216 (2004), no. $1,149-175$.

[9] M. SPIVAK, A comprehensive introduction to differential geometry, Vol. V, Publish or Perish, Inc., 1979.

[10] M. UMehara and K. Yamada, Complete surfaces of constant mean curvature 1 in the hyperbolic 3-space, Ann. of Math. (2) 137 (1993), no. 3, 611-638.

Present Address:

DePARTMENT OF MATHEMATICS, SCHOOL OF ENGINEERING,

TOKYO DENKI UNIVERSITY,

KANDA-NISHIKI-CHO, CHIYODA-KU, TOKYO, 101-8457 JAPAN.

e-mail: kokubu@cck.dendai.ac.jp 